\title{
Using simulations to explore sampling distributions: an antidote to hasty and extravagant inferences
}

\author{
Guillaume A. Rousselet ${ }^{1 *}$ \\ ${ }^{1}$ Institute of Neuroscience \& Psychology, University of Glasgow, Scotland \\ *Email: Guillaume.Rousselet@glasgow.ac.uk
}

\begin{abstract}
Most statistical inferences in psychology are based on frequentist statistics, which rely on sampling distributions: the long-run outcomes of multiple experiments, given a certain model. Yet, sampling distributions are poorly understood and rarely explicitly considered when making inferences. In this article, I demonstrate how to use simulations to illustrate sampling distributions to answer simple practical questions: for instance, if we could run thousands of experiments, what would the outcome look like? What do these simulations tell us about the results from a single experiment? Such simulations can be run a priori, given expected results, or a posteriori, using existing datasets. Both approaches can help make explicit the data generating process and the sources of variability; they also reveal the large variability in our experimental estimation and lead to the sobering realisation that, in most situations, we should not make a big deal out of results from a single experiment. Simulations can also help demonstrate how the selection of effect sizes conditional on some arbitrary cut-off $(p \leq 0.05)$ leads to a literature crammed with false positives, a powerful illustration of the damage done in part by researchers' over-confidence in their statistical tools. The article focuses on graphical descriptions and covers examples using correlation analyses, percent correct data and response latency data.
\end{abstract}

Keywords: correlation, reaction times, ERP, power, false positives, precision, estimation, sample size 


\section{Introduction}

The typical psychology or neuroscience article reports data from one or a very few experiments. Inferences are usually made about some unspecified population using frequentist statistics. An arbitrary cut-off is then used to dichotomise effects as significant or not, and to claim a discovery, irrespective of other sources of information (McShane, Gal, Gelman, Robert, \& Tackett, 2019). This mostly mindless ritual gives the illusion of certainty despite the noise and variability inherent to data collection and analysis (Gelman, 2018; Gigerenzer \& Marewski, 2015; Yarkoni, 2019). A large part of the so-called replication crisis is probably due to this over-confidence, stemming from the erroneous belief that statistical methods can deliver the truth. In practice, most discoveries can only be made in the long-run, following an extensive programme of research (Morey, 2018). This long-run perspective is often lost in the flashy short papers that claim a discovery based on one noisy sample. It is therefore essential for researchers to be aware of this long-run perspective. One of the most efficient ways to achieve this goal is to perform simulations. Here we look at sampling distributions to consider results not from one experiment but from thousands of them. This can be done using synthetic or real data, and the resulting sampling distributions can be used to answer useful questions. In this article, after defining sampling distributions, I propose a series of examples exploring estimation variability across simulated experiments. We will consider analyses of correlations, proportion correct data and two types of response latency data. The examples are relatively simple, but they help demonstrate the potentially large benefits of learning to and spending the time to write a few lines of code to simulate experimental results. By engaging in this type of exercise, we have to explicitly consider the data generating process, including the experimental design and the shape of the population distributions we sample from when we carry out experiments. We also directly visualise the effect of sample sizes. As a result, the simulation process should ultimately lead to better planning of experiments and also a better understanding and acceptance of uncertainty, a more modest interpretation of results, and a healthy scepticism of published results. 


\section{Sampling distributions}

Sampling distributions are at the core of inferential frequentist statistics. Indeed, frequentist statistics deal with the long run outcomes of imaginary experiments. For instance, when performing a $t$-test, we use the sampling distribution of $t$ values assuming that there is no effect to compute the probability of observing a $t$ value at least as large as the one we obtained based on our sample-the so-called $p$ value (Greenland et al., 2016). So the point is that all users of frequentist statistics already use sampling distributions, although they might not know it. But there is more to sampling distributions than $t$-tests and other standardised statistics. Sampling distributions can be used to learn about the long-run behaviour of certain quantities over many experiments. For instance, we can ask what we can expect to observe if we did thousands of experiments.

A sampling distribution is essentially the outcome of a simulation in which the same experiment is carried out many times (Baguley, 2012). To illustrate, let's imagine that we perform experiments in which we sample from the skewed distribution in Figure 1A. This distribution has mean $\mu=1.13$ and standard deviation $\sigma=0.604$, and like most quantities we measure in psychology, it takes only positive values. From these population parameters, the standard error of the mean (SEM) is defined as $\sigma / \sqrt{n}$; thus 0.191 for $\mathrm{n}=10,0.135$ for $\mathrm{n}=20,0.085$ for $\mathrm{n}=50$. The $\sigma$ population value is not usually observable, so it is estimated from random samples of observations (aka experiments), using the sample standard deviation $s d$, such that $S E M=s d / \sqrt{n}$. 


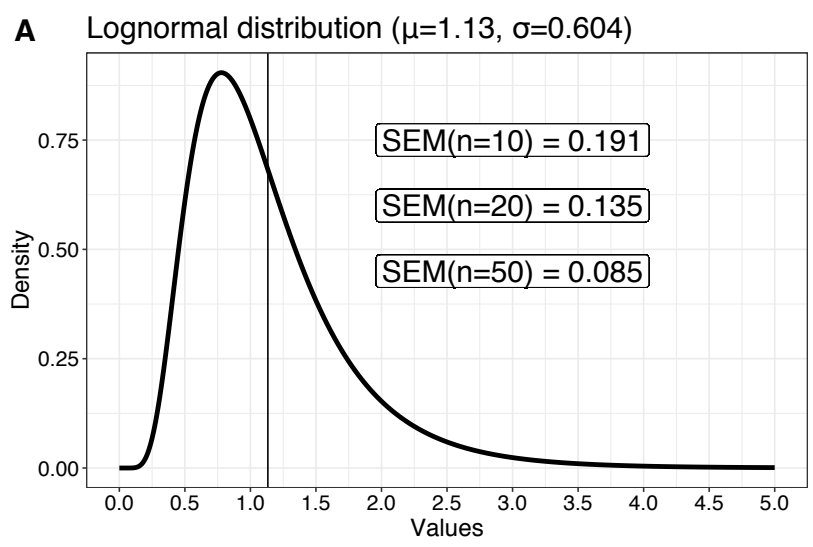

B Random samples of $\mathrm{n}=10$ trials

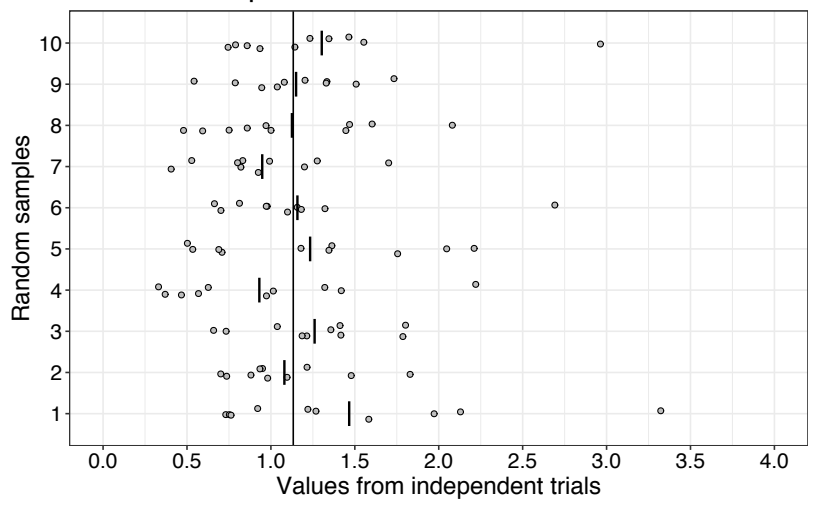

C Sampling distributions

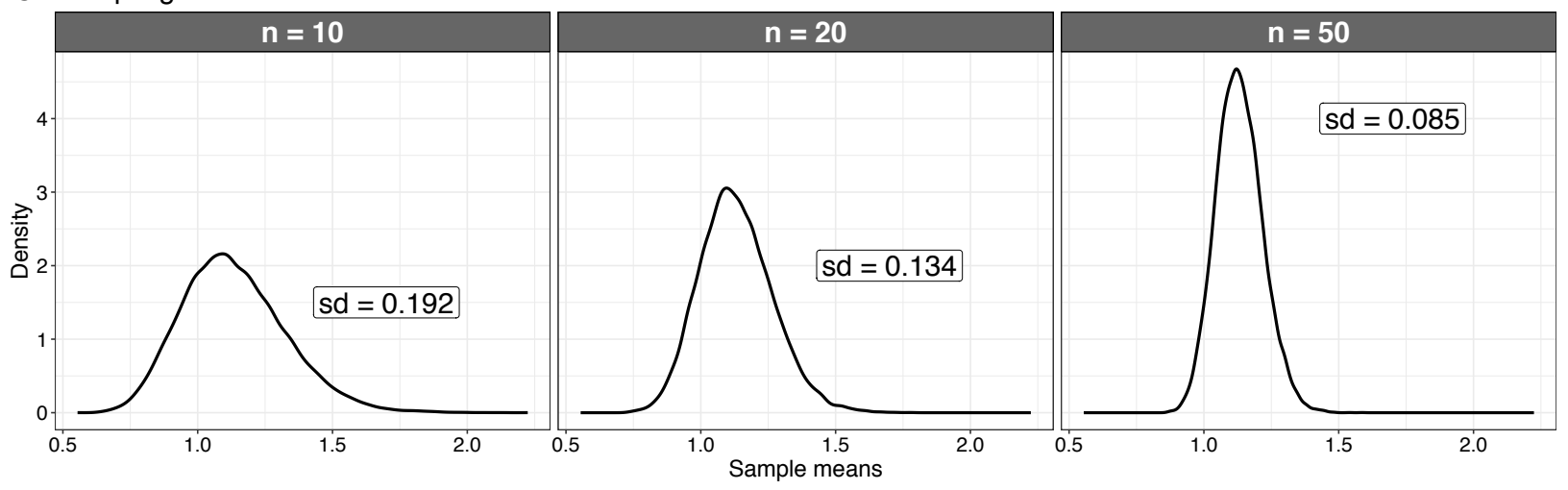

Figure 1. Sampling from a lognormal distribution. A. Lognormal distribution with parameters $\log \mu=0, \log$ $\sigma=0.5$. The corresponding population mean and standard deviation are $\mu=1.13, \sigma=0.604$. The vertical line marks $\mu$. The insets contain the standard errors of the mean (SEM) for three sample sizes. B. Scatterplots of 10 random samples from A. The long vertical line marks the population mean. Each disk is an observation. The short vertical lines mark the sample means. C. Sampling distributions of the mean for 20,000 samples of sizes $n=10, n=20, n=50$ observations. For each sample size, the standard deviation of the sampling distribution of the sample mean is equal to the SEM. This figure was created using the R notebook lognormal.Rmd.

Random samples of 10 observations are illustrated in Figure 1B. Each of these samples has a different sample mean, a different sample $s d$, and therefore a different estimated SEM. This seems obvious, but panel B reminds us that there can be a lot of variability across experiments measuring the same phenomenon, something that is easy to forget when considering the outcome of a single experiment.

Instead of a few virtual experiments, we can perform many of them to see how the sample mean is distributed in the long run. Figure $1 \mathrm{C}$ shows the outcome of 20,000 experiments in which we randomly sample n observations from a standard lognormal population (Figure 1A) and compute 
the sample mean. The standard deviations of these sampling distributions are equal to the population SEMs (compare insets in panels A and C - the small differences are due to limited number of simulation iterations). This is because the two quantities are the same. The SEM is simply the SD of the sampling distribution of the sample mean ${ }^{1}$. Hence, whereas the sample $s d$ tells us about the variability of observations about the sample mean, SEM tells us about the variability of the sample mean about the population mean. This important point is discussed in any decent introductory frequentist statistics textbook (e.g. Howell, 2013).

We can also make two important observations from the sampling distributions. First, they are skewed, even for $\mathrm{n}=50$, which violates the assumption of the $t$-test, leading to inaccurate $p$ values and confidence intervals (Rousselet, Pernet, \& Wilcox, 2019; Wilcox \& Rousselet, 2018). Second, with increasing sample size, the distributions get narrower, which means that each sample mean is on average closer to the population mean. And that's the main reason to use large sample sizes: to get closer to the truth. Hence, computing sampling distributions and illustrating them can provide intuitive descriptions of the long-run behaviour of a quantity, from which we can assess if the assumptions of statistical tests are met and help us grasp how far off our experimental results could be from the truth. In the rest of this article, we focus on the second aspect by exploring sampling distributions in a series of examples, looking at analyses of correlations, proportion correct data and response latency measurements.

\section{Correlation analyses}

Although correlation analyses may not be the optimal way to answer the most meaningful questions about one's data (Baguley, 2009, 2010), they are omnipresent in the literature. Like the more flexible and meaningful regression analyses, correlation analyses suffer from two major issues. First, standard techniques are not generally robust to violations of their assumptions. Such violations can considerably affect the estimation of correlation and regression coefficients (Wilcox, 2017). Second, the sample size strongly affects the precision of the estimates, and small

1 This video explains clearly sampling distributions and the SEM: https://www.youtube.com/watch?v=J1twbrHel3o 
sample sizes may yield highly inaccurate estimates. Here we focus on the second issue as it pertains to correlation analyses.

The problem with small sample sizes in correlation analyses is not new. About 10 years ago, Vul et al. warned the community about the prevalence of false positives in brain-behaviour correlation analyses. They argued that standard practices led to so-called voodoo correlations (Vul, Harris, Winkielman, \& Pashler, 2009a, 2009b). In one of several replies to their article, Yarkoni suggested that the main cause of voodoo correlations was the lack of power of studies with small sample sizes (Yarkoni, 2009). Yarkoni's argument is this: correlation estimates from small samples are imprecise, such that even if samples are taken from a population with a zero correlation, large correlation estimates can be expected by chance. However, in small samples, only extreme correlation estimates yield statistically significant $(p \leq 0.05)$ tests. This lack of power, combined with publication bias towards new, unexpected positive results, can easily lead to a literature replete with overestimates (Forstmeier, Wagenmakers, \& Parker, 2016). Although this problem is well documented, in my experience, it is still very common to see articles with sample sizes too small to estimate correlations with sufficient precision.

Let's illustrate the problems associated with small sample sizes by looking at sampling distributions. But first, let's start with the example in Figure 2: nice scatterplot isn't it?!

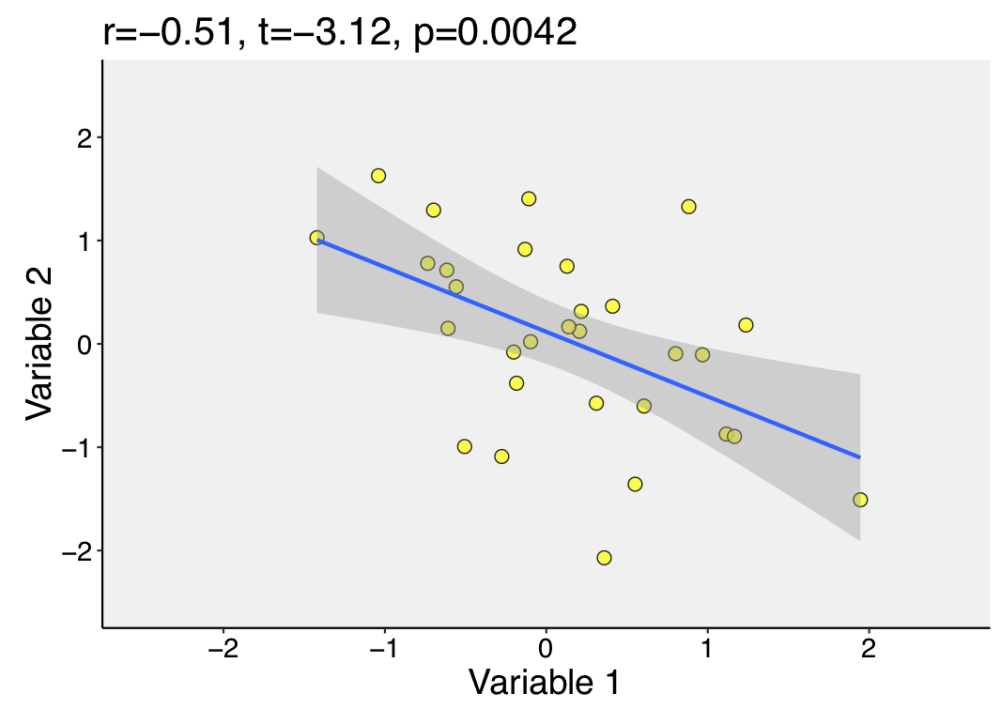

Figure 2. Nice looking but random correlation? The sample size is $n=30$. The bivariate sample comes from a population with a true effect size $\rho$ of 0 . In other words, we are looking at sampling noise. This figure was created using the R notebook corr_sim.Rmd. 
Sample size is 30, and the estimated Pearson's correlation coefficient $r$ is -0.51 . It seems we have discovered a relatively strong association between variables 1 and 2! Unfortunately, this effect will not replicate, because the bivariate data in the scatterplot were sampled from a population with zero correlation ( $\rho=0$, the Greek letter $\rho$ represents the population correlation coefficient). So the true effect is zero, but our sample leads us to believe otherwise. There is nothing new here: inflated effect sizes are a natural outcome of studies with small sample sizes. The problem only gets worse once we add questionable research practices (such as selective reporting) and incentives to publish novel, positive results to the equation. (To be fair, the example in Figure 2 is the outcome of selective reporting: I generated all the correlations among 20 samples and picked the samples associated with the highest correlation to make a point!)

The effect size inflation with small sample sizes might seem counter-intuitive because, if a study lacks power, surely the true effect must be very strong to show up with such a small sample size. However appealing, this conclusion is wrong because it ignores a critical aspect of studies with small sample sizes: they are associated with large sampling variability, which means that estimation precision is poor. This error in reasoning has been described as the "which does not kill statistical significance makes it stronger" fallacy (Loken \& Gelman, 2017). The problem becomes clear when we draw samples of different sizes from a normal bivariate population with a known population Pearson correlation $\rho$ of 0 . The sampling distributions of the estimates of $\rho$ for different sample sizes are shown in Figure 3A, which illustrates that the sample estimates often differ considerably from the population value, particularly in small samples. 

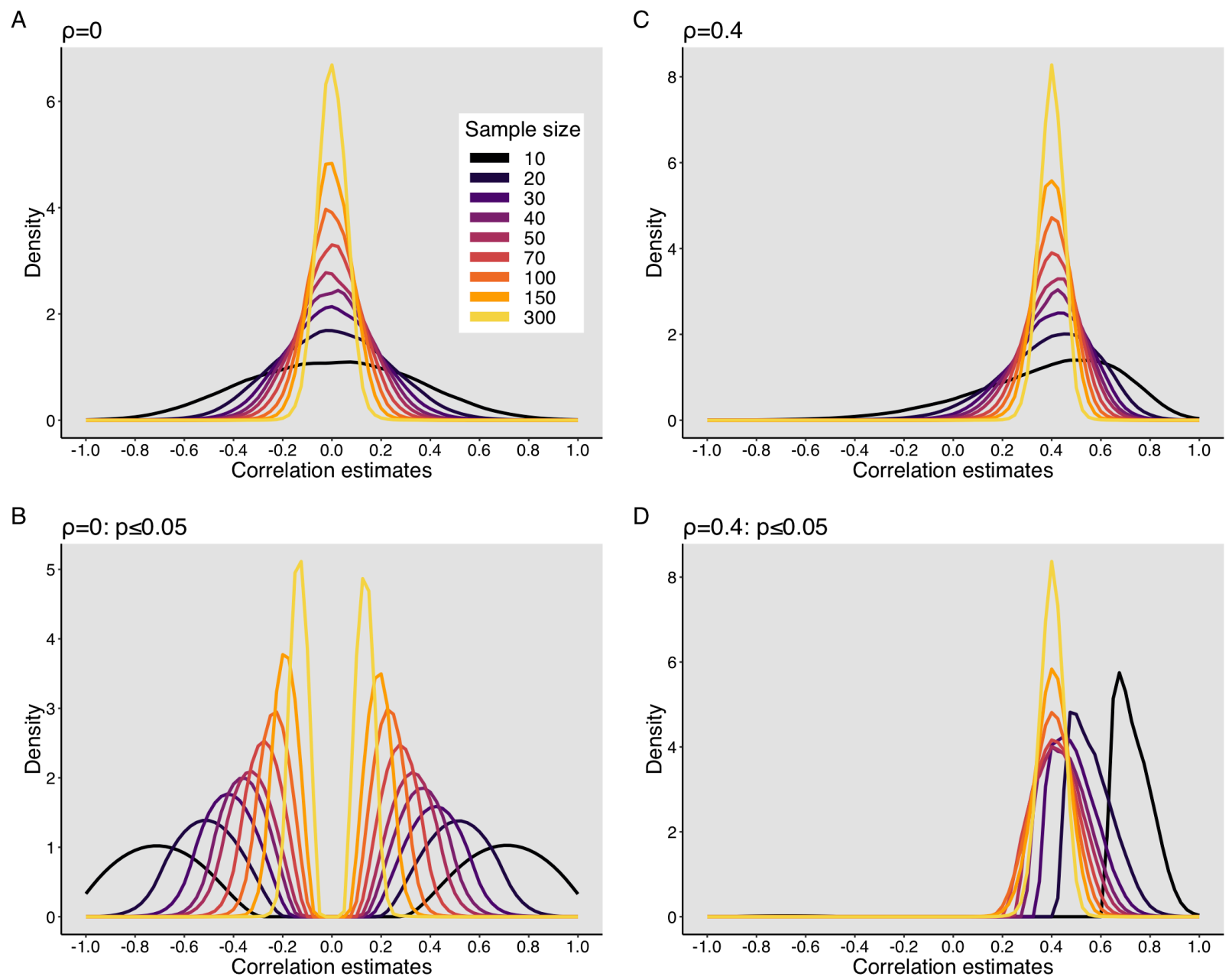

Figure 3. Examples of sampling distributions of correlation coefficients. Results are presented for Pearson's correlation. Similar results would be obtained using Spearman's correlation or some other measure of association. A. Sampling distribution for $\rho=0$. B. Sampling distribution for $\rho=0$, given $p \leq 0.05$. C. Sampling distribution for $\rho=0.4$. D. Sampling distribution for $\rho=0.4$, given $p \leq 0.05$. This figure was created using the $\mathrm{R}$ notebook corr_sim.Rmd.

Sampling distributions tell us about the behaviour of a statistic in the long-run, if we did many studies. With larger sample sizes, the sampling distributions are narrower, which means that the individual estimates tend to be more precise. However, a typical article reports only one correlation estimate, which could still be completely off. So what sample size should we use to get a precise estimate? The answer depends on:

- the shape of the univariate and bivariate distributions;

- the true effect size (the larger the effect, the fewer trials are needed-see below); 
- the precision we want to afford.

For the sampling distributions in Figure 3A, we can calculate the proportion of correlation estimates that are within a certain margin from the population correlation $(\rho=0)$. For instance, as shown by the black arrows in Figure 4A:

- for $70 \%$ of estimates to be within 0.1 of the true correlation value (i.e., between $r=-0.1$ and 0.1 , we need at least 110 observations;

- for $90 \%$ of estimates to be within 0.2 of the true correlation value (i.e., between $r=-0.2$ and 0.2), we need at least 69 observations.

Of course, the values I used above are completely arbitrary. Why $70 \%$ ? Why \pm 0.1 ? This is not different from any thresholds used in statistics: for instance, what's the rational for $\mathrm{p} \leq 0.05,95 \%$ confidence intervals, $80 \%$ power, Bayes factor $>3$, and the inane trichotomisation of Cohen's $d$, other than habit? Providing multiple curves, as in Figure 4A, at least let different researchers consider different thresholds, based on different considerations and their knowledge of the literature.

The bottom line is that even if we're willing to settle for fairly imprecise estimates (up to 0.2 from a true value of 0 ), we need plenty of observations to achieve this precision often enough - again, there is no guarantee whatsoever for any particular experiment we conduct. This approach fits well with a growing literature that advocates planning experiments for estimation precision rather than the traditional goal of power (Bland, 2009; Gelman \& Carlin, 2014; Maxwell, Kelley, \& Rausch, 2008; Peters \& Crutzen, 2017; Rothman \& Greenland, 2018; Schönbrodt \& Perugini, 2013; Trafimow \& MacDonald, 2017; Trafimow, Wang, \& Wang, 2019).

Not surprisingly, tests based on small sample sizes also lack statistical power as shown in Figure 4B. Statistical power (or proportion of true positives) refers to the probability to detect an effect using an a priori cut-off (typically $\mathrm{p} \leq 0.05$ ) when an effect exists. For instance, assuming samples from a bivariate normal distribution: 
- to achieve $80 \%$ power given a true population correlation of $\rho=0.4$, the required sample size is 46 observations;

- to achieve $90 \%$ power given a true population correlation of $\rho=0.3$, the required sample size is 118 observations.

Alternatively, for a given sample size and a desired power, we can determine the minimum effect size we can hope to detect. For instance, with a sample size of $n=40$, the minimum true effect size we can hope to detect with $90 \%$ power is $\rho=0.49$.

A

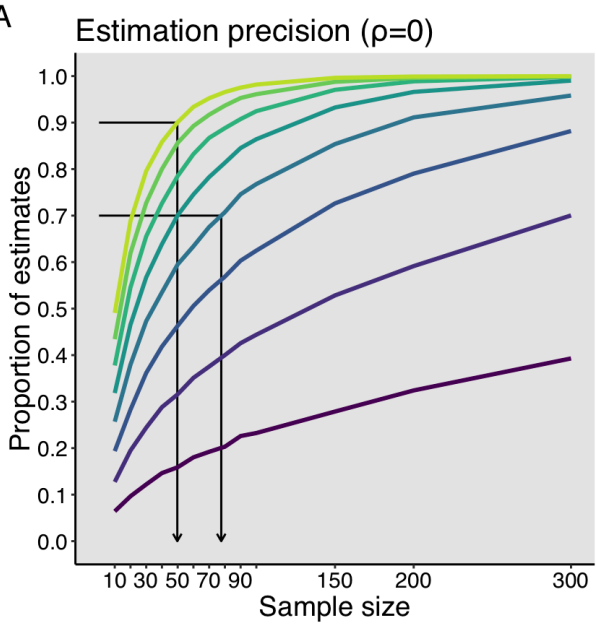

B

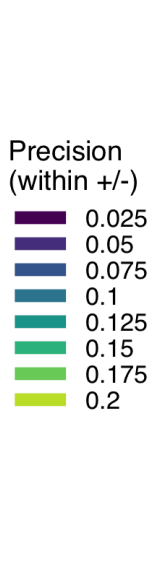

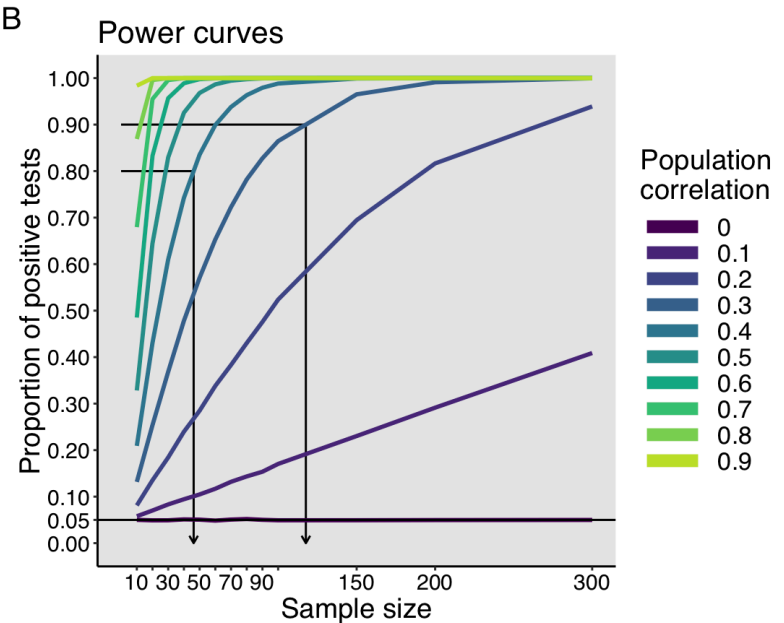

Figure 4. Correlation: estimation precision and power curves. A. Proportions of estimates near the true value $(\rho=0)$, for different sample sizes, and for different levels of precision. B. Proportions of tests with $p \leq 0.05$ (power) for different sample sizes and population correlations $(\rho)$. This figure was created using the $\mathrm{R}$ notebooks corr_sim.Rmd and corr_power.Rmd.

However, estimating statistical power when planning experiments is difficult because the literature almost certainly provides a biased estimation of the true population correlations (Gelman \& Weakliem, 2009; Ioannidis, 2008). Indeed, consider what happens to a literature in which it is exceedingly difficult to publish non-significant findings (Smaldino \& McElreath, 2016). We can illustrate this problem by looking at the sampling distribution of only those correlation coefficients that happen to yield statistical significance $-p \leq 0.05$ (Figure 3B). 
For a correlation estimate to be significant, its absolute value needs to be fairly large--more so for small samples. Selecting for a significant correlation coefficient therefore removes the part of the sampling distribution around $r=0$ and only retains the more extreme values. If authors, reviewers, and editors are biased against non-significant findings, this means that readers only get to see an upwardly biased portion of the total distribution of correlation estimates. This, in turn, means that if researchers base their power analyses on the effect sizes encountered in the literature, they are likely to overestimate their targeted effect size - and hence the power of their programme of research.

So far, we have considered samples from a population with zero correlation, such that any large positive or negative correlations were due to chance. Let us see what happens when there is a non-zero effect for a fixed sample size of 30 . As Figure 5 shows, the modes of the sampling distributions increase with increasing population correlations, whereas their spreads decrease.

\section{Correlation sampling distributions $(n=30)$}

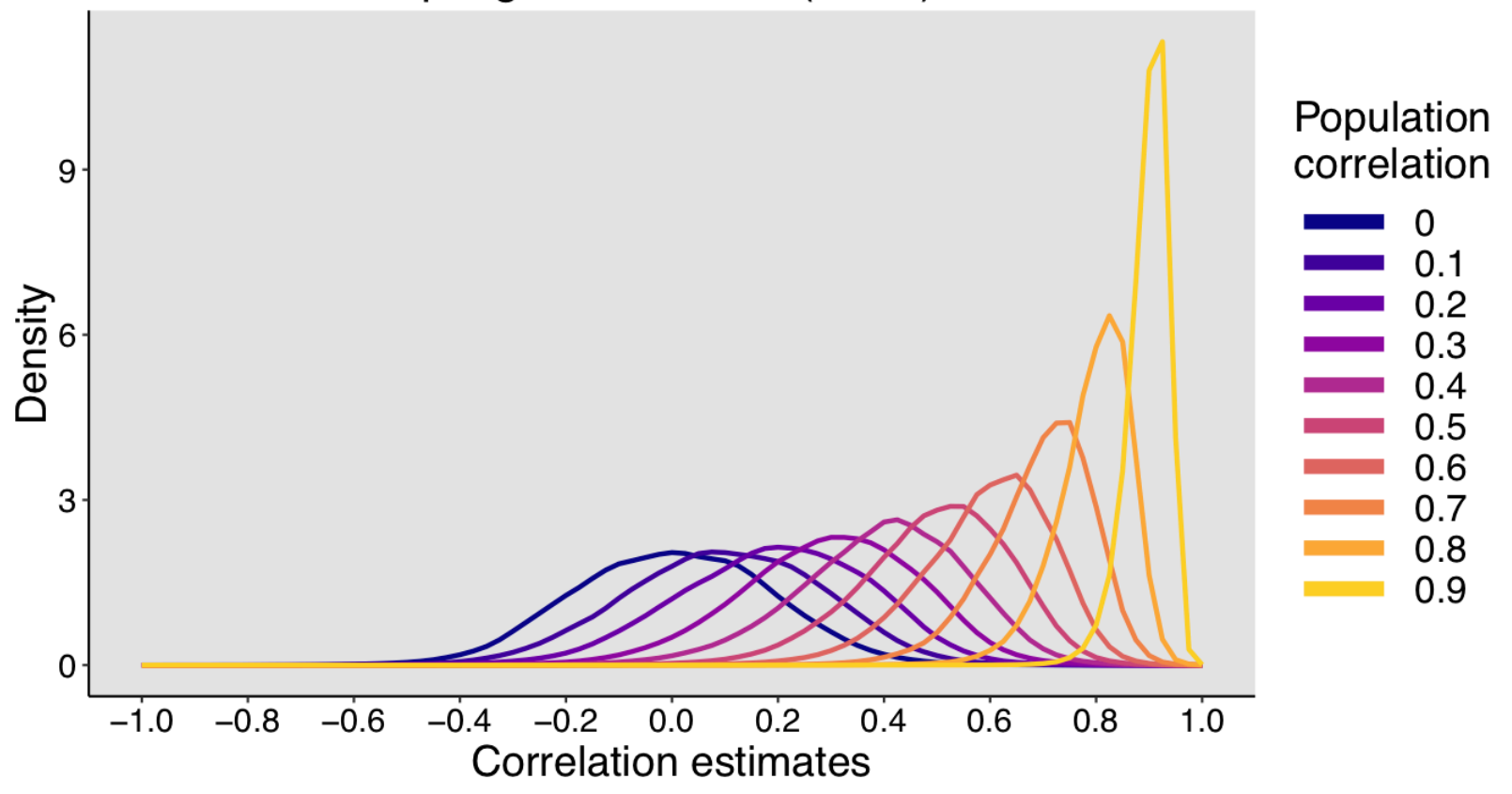

Figure 5. Correlation sampling distributions as a function of the population correlation. The sample size is always $\mathrm{n}=30$. This figure was created using the $\mathrm{R}$ notebook corr_sim.Rmd.

Consider in more detail the sampling distributions for $\rho=0.4$ (Figure 3C). The sampling distributions for $\mathrm{n}<50$ are negatively skewed. Consequently, the typical experiment will tend to 
overestimate the true value (distribution mode shifted to the right). If we report only correlations associated with $p \leq 0.05$, the distributions look very different (Figure 3D). Again, with small sample sizes, the estimates are inflated, albeit in the correct direction. There is nevertheless a small number of large negative correlations: indeed, in $0.77 \%$ of simulations, even though the population value was 0.4 , a large and $p \leq 0.05$ negative correlation was obtained-also known as a directional or type III error (Shaffer, 2002).

The main lesson from this section is that the minimum sample size needed to estimate a particular association is probably much larger than we tend to think. And this is not just a point about statistics, because the size of the true correlations we can expect in real data is also probably much weaker than often reported based on small sample sizes. For instance, large $\mathrm{n}$ studies and meta-analyses in the social sciences suggest that correlations between 0.2 and 0.4 should be considered relatively large, and weaker correlations are much more common (Frey, Pedroni, Mata, Rieskamp, \& Hertwig, 2017; Plonsky \& Oswald, 2014; Schönbrodt \& Perugini, 2013), with some replication attempts demonstrating much smaller effect sizes when using larger samples than in the original studies (e.g. Cai et al., 2019; Zhang et al., 2018). Also, because the literature is biased towards positive results, meta-analyses tend to over-estimate the population effect sizes. In brain imaging, a recent study estimated correlations between resting-state fMRI data and personality traits across 884 participants (Dubois, Galdi, Han, Paul, \& Adolphs, 2018). The largest $r$ was 0.27 . Also, some of the results were highly susceptible to preprocessing methods, an analytical flexibility that could be exploited to find and only report the best possible outcome, leading to inflated estimates in the literature. In another study involving 5,216 subjects, correlations between several brain structural measurements and two cognitive tasks were all inferior to 0.20 (Ritchie et al., 2018). And a meta-analysis of 88 studies (8036 subjects) of correlations between brain volume and IQ suggests an overall effect of $r=0.24$ (Pietschnig, Penke, Wicherts, Zeiler, \& Voracek, 2015). Thus, in the absence of large-sample replications, large correlations are best taken with a grain of salt. There are, however, a few notable exceptions where large effect sizes are expected, such as test-retest assessment and the comparison of related tests or measurements. 


\section{Percent correct data}

By looking at sampling distributions of correlation coefficients, we learnt important lessons about the relationship between estimation precision and sample size, and how conditioning on arbitrary cut-offs can dramatically bias effect sizes in the literature. We now turn our attention to another type of popular analyses, that of proportion correct data. This will again help us illustrate the importance of sample sizes, but also highlight another important aspect of simulations: to be explicit about the data generating process.

Let's consider the simulated data in Figure 6. Panel A illustrates the theoretical relative probabilities of observing different proportions of correct responses for different true values. For instance, given that a participant is on average $10 \%$ correct, what is the distribution of the number of correct trials we can expect across many experiments with 100 trials? For any given experiment, the number of correct trials ranges from roughly 0 to 20 . If the population average is $50 \%$, the values across experiments range roughly from 35 to 65 . Thus, the population mean percent correct and standard deviation are dependent: there is stronger variability at $50 \%$ correct and the variability decreases as the mean tends toward zero or 1.

Although this type of data are best analysed using hierarchical (mixed-effect) models (Jaeger, 2008; Kruschke, 2014), in psychology correct/incorrect results tend to be averaged across trials for each condition and participant, before entering the averages into an ANOVA. What do the sampling distributions across participants look like? Because participants cannot be less than 0\% correct or more than $100 \%$ correct, the distribution must be bounded, which rules out any continuous distribution such as the normal distribution as a good model-yes, standard analyses assume that participants can be more than 100\% correct! Instead, a good candidate is a beta distribution, which is bounded between 0 and 1 (Kruschke, 2014). Figure 6B shows an example of a beta sampling distribution with a mean of $70 \%$ correct. The distribution is negatively skewed and quite broad. Using this beta distribution, we can simulate the sampling variability expected in an experiment: each sample from the distribution corresponds to the proportion correct of a participant; then for that value, we generate trials from a binomial distribution. Figure 6C illustrates 10 random samples of 10 participants. For each participant, a random value from the 
beta distribution determined their proportion of correct trials and 100 trials were generated by sampling from the corresponding binomial distribution. Again, these random samples help us grasp the large variability inherent to experimental sampling. 
A Binomial PDFs for $n=100$ trials

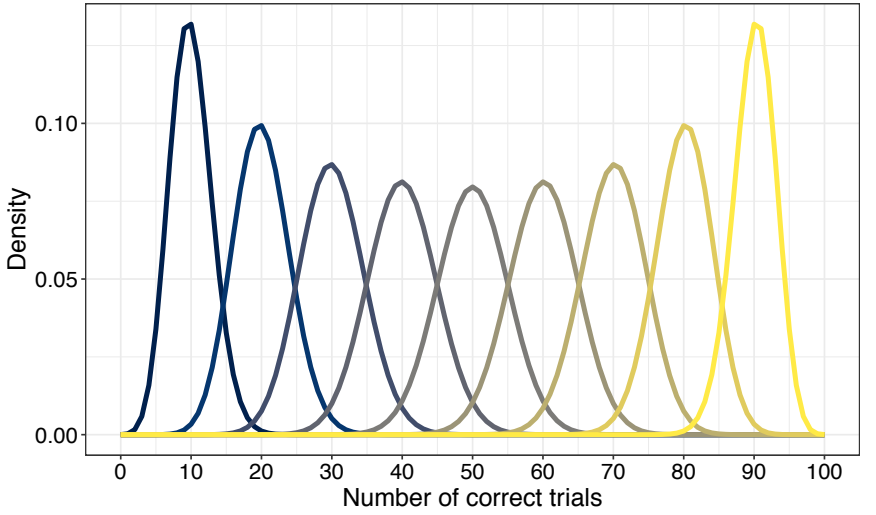

B Beta distribution: alpha $=20$, beta $=8.56$, mean $=0.7$

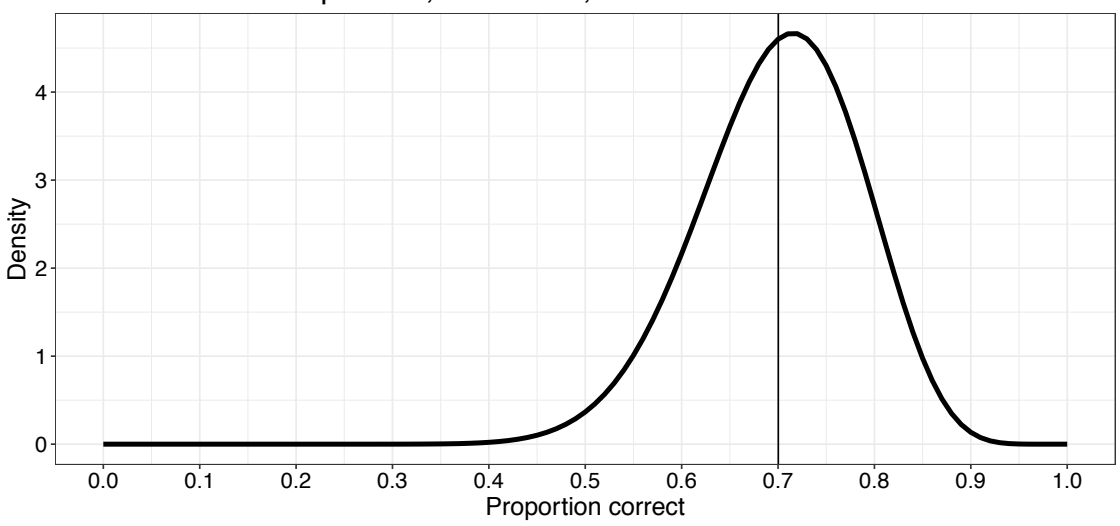

C Random samples: 10 participants, 100 trials
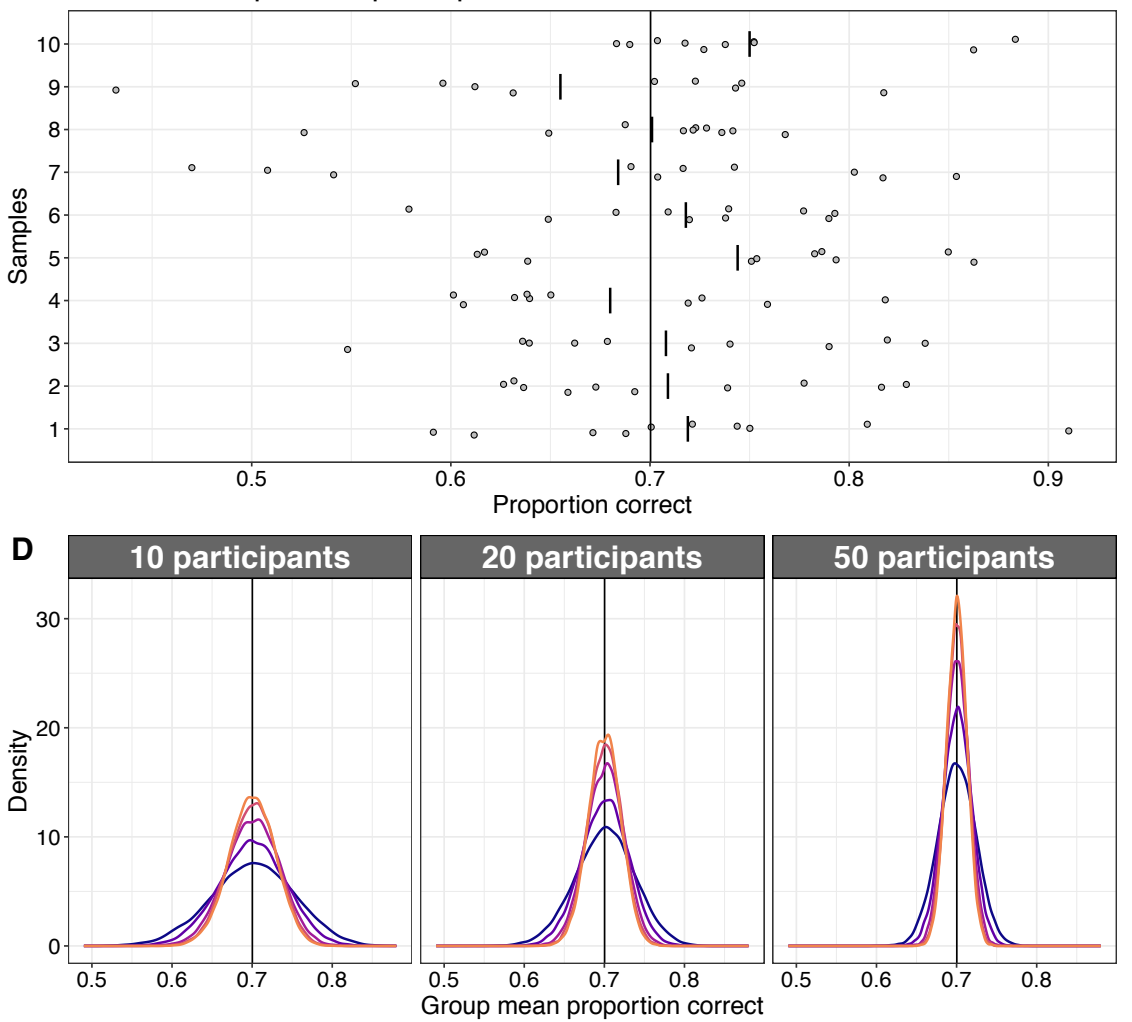

Number of trials

10

Population proportion correct

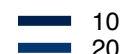

30

40

50
60

$-\quad 70$
$-\quad 80$

80

-90
-10

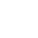


Using the same approach, we can perform 20,000 simulated experiments in which we vary the number of participants and the number of trials per participant. The results are presented in Figure 6D. As illustrated in our correlation examples, with increasing sample sizes, precision increases (sampling distributions get narrower). The results also suggest something very useful to plan experiments: the precision increases faster with the number of participants than with the number of trials (compare panel 10 to panel 50). For instance, with 10 participants and 100 trials per participant, the probability to observe a group mean more than $5 \%$ points from the population mean is about $10 \%$. With only 50 trials per participant, this probability is now $14 \%$. But if we test 50 participants with 10 trials each, the probability is now only $3.5 \%$, even though both situations involve a total of 500 observations. The increased benefit of participants over trials seem to apply to many situations (Rouder \& Haaf, 2018).

\section{Response latencies}

\section{ERP onsets}

Besides proportion correct data, another popular type of data are measures of response latencies. Here we consider two examples, one using event-related potential (ERP) data, one using manual reaction time data. All such latency data are bounded and skewed, so it is important to consider sampling distributions.

In our first example, ERP onsets (earliest differences between conditions) were estimated in 120 participants engaged in a face vs. texture discrimination task; 74 of them were tested in a second session to assess test-retest reliability (Bieniek, Bennett, Sekuler, \& Rousselet, 2016). Here for convenience we merge the two sessions to form a distribution of 194 ERP onsets, which is

positively skewed (Figure 7A). Because the typical ERP experiment has much fewer participants, we can use data-driven simulations to determine sampling distributions given smaller sample sizes. If we are interested in the central tendency of a skewed distribution, it can be informative to estimate the 50th quantile of the distribution-the value that splits the distribution of sorted observations in two equal parts (Rousselet \& Wilcox, 2019). To estimate the sampling distributions of the $50^{\text {th }}$ quantile, given our data, we sample from the distribution of onsets 
10,000 times, using sample sizes from 10 to 40, incremented in steps of 5 . The resulting distributions are presented in Figure 7B.
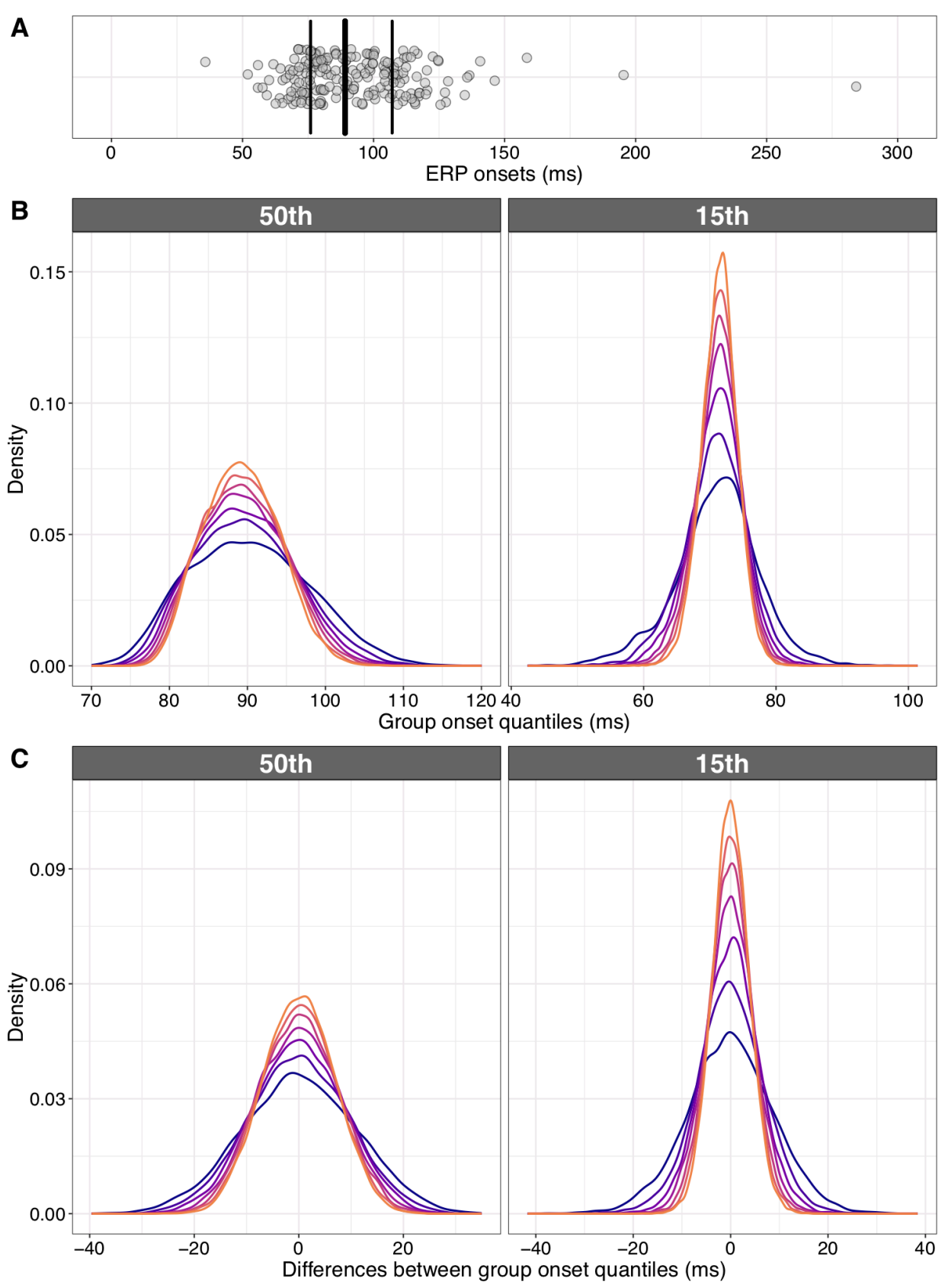

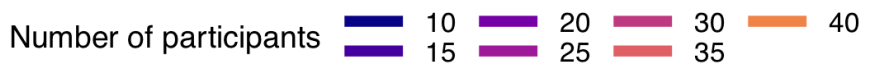

Figure 7. ERP

onsets. A.

Scatterplot of 194

ERP onsets. The

vertical lines mark the

quartiles. B.

Sampling

distributions of the

50th (left) and the

15th (right) onset

quantiles. The

quantiles were

computed using the

Harrell-Davis

estimator, which deals better with tied values than standard estimators (Harrell \& Davis, 1982). The results are based on 10,000 simulated experiments. C.

Sampling

distributions of quantile differences

between two experiments. This figure was created using the $\mathrm{R}$ notebook onsets.Rmd. 
From these distributions, we can ask useful questions by treating the full sample as a population. For instance, what is the proportion of onsets estimates that are within $+/$ - a certain value from the population onset quantile?

Here we can motivate our choice of threshold value using the neuroscience literature. For instance, it has been estimated that on average, for short latency neurones, the transfer time between cortical areas is about $10 \mathrm{~ms}$ (Nowak \& Bullier, 1997). So when looking at fast visual responses, $10 \mathrm{~ms}$ seems like a meaningful level of precision we should care about.

So let's go with $10 \mathrm{~ms} .$. With a sample size of 10 participants, about $80 \%$ of 50 th quantile estimates are within $+/-10 \mathrm{~ms}$ of the population value. With a sample size of 35 participants, about $97 \%$ of 50 th quantile estimates are within $+/-10 \mathrm{~ms}$ of the population value. We can also determine the number of trials needed to achieve a certain level of estimation precision. For instance, to be within $10 \mathrm{~ms}$ of the full sample 50th quantile value in at least $90 \%$ of experiments, how many participants do we need? The answer is $n=19$ participants.

There is no need to restrict our investigation to one quantile only. If the focus is on processing speed, it is of particular interest to quantify the fastest responses. This can be done for instance by estimating a lower quantile, say the 15 th quantile (Figure $7 \mathrm{~B}$ ). You could pick the $17^{\text {th }}$ quantile if you have a preference for prime numbers, for instance.

Given the controversies about the latencies of the first face responses in the brain, it can be useful to perform a simulation in which we quantify, given our data, how far apart estimates can be between two experiments (Figure 7C). We can determine, given a certain level of precision, the probability to observe similar effects in two experiments. For instance, with a sample size of 10 participants, only about $63 \%$ of 50 th quantile estimates are within $+/-10 \mathrm{~ms}$ of each other. With a sample size of 35 participants, about $86 \%$ of 50th quantile estimates are within $+/-10 \mathrm{~ms}$ of each other. Conversely, we can determine the number of observations needed to achieve a certain performance. For instance, for $90 \%$ of pairs of experiments to generate results at most 15 ms apart, we need at least 18 observations. Such calculations might suggest that certain 
discrepancies in the literature are simply due to random sampling fluctuations, or might point to real differences due to particular factors, such as the task or the age of the participants. Whatever the reason, simulations of sampling distributions help put results in perspective.

\section{Reaction time data}

The previous example considered a situation with only one level of analysis: for simplicity we did not consider within-participant variability because of the large amount of data involved in computing ERP onsets in each participant. Here we use a dataset containing a large number of participants and a large number of trials for each participant. The data are from the French Lexicon Project (Ferrand et al., 2010): manual reaction times where measured in response to word and non-words. After discarding participants who clearly did not pay attention, we are left with 967 participants, each with about 1000 trials per condition (no further data cleaning was

performed, such as removing outlier trials). Examples of individual reaction time distributions are shown in Figure 8A. The distributions are positively skewed, as expected for RT data, and participants tend to be slower in the Non-Word condition compared to the Word condition. Usually, a single number is used to summarise each individual RT distribution-a better alternative would be to use a hierarchical model including stimulus and participant effects (Rouder \& Province, 2019; Yarkoni, 2019). From 1,000 values to 1, that's some serious data compression! In psychology, the mean is often used, but when there is skewness it is an inappropriate measure of location (Rousselet \& Wilcox, 2019; Trafimow, Wang, \& Wang, 2018). So here we use the $20 \%$ trimmed mean, which gives a better indication of the location of the typical observation and protects against the influence of outliers (Wilcox, 2017). To compute a $20 \%$ trimmed mean, we sort the data, remove the $20 \%$ lowest values and the $20 \%$ highest values and average the remaining values. In that context, the median is a 50\% trimmed mean and the mean is a $0 \%$ trimmed mean. We could trim more or less, but $20 \%$ works well in many situations (Wilcox, 2017) and this particular choice is irrelevant to the points I'm about to make.

The distributions of participants' $20 \%$ trimmed means are positively skewed in both conditions (Figure 8B). And because these distributions differ in skewness, the distribution of differences between the $20 \%$ trimmed means in the two conditions is also skewed (Non-Word minus Word). 
The distribution of differences overlaps very little with zero: $96.2 \%$ of participants have a positive difference, meaning that they tended to be faster in the Word than the Non-Word condition. With this large dataset, as in previous examples, we can pretend that the full dataset is our population that we're trying to estimate. We perform data-driven simulations to get sampling distributions. In this example, the sampling is hierarchical: we sample with replacement participants, and for each randomly sampled participant, we sample trials with replacement. Because there is skewness at both levels of analysis, we compute 20\% trimmed means across trials for each condition and then compute 20\% trimmed means across participants. This was done for simulations with 5,000 iterations in which we varied independently the number of participants and the number of trials (Figure 8C).

Figure 8. Reaction time data from the FLP dataset. A. Examples of individual reaction time distributions from 20 participants, in the two conditions. B. Distributions of 20\% trimmed means in 967 participants: for each participant, a 20\% trimmed mean was computed across trials to summarise the distribution in each condition. The right panel shows the distribution of differences between 20\% trimmed means in the two conditions (Non-Word minus Word). Note how most of the differences are positive, but with very large variability. G. Sampling distributions for the group 20\% trimmed means, based on simulations with 5,000 iterations. The vertical line in each panel marks the population $20 \%$ trimmed mean. D. Sampling distributions when there is no effect. This was simulated by pooling the two conditions together. The first row illustrates the sampling distributions as they are (raw data); the second row illustrates the same results after conditioning on $p \leq 0.05$. This figure was created using the $\mathrm{R}$ notebook flp.Rmd. 

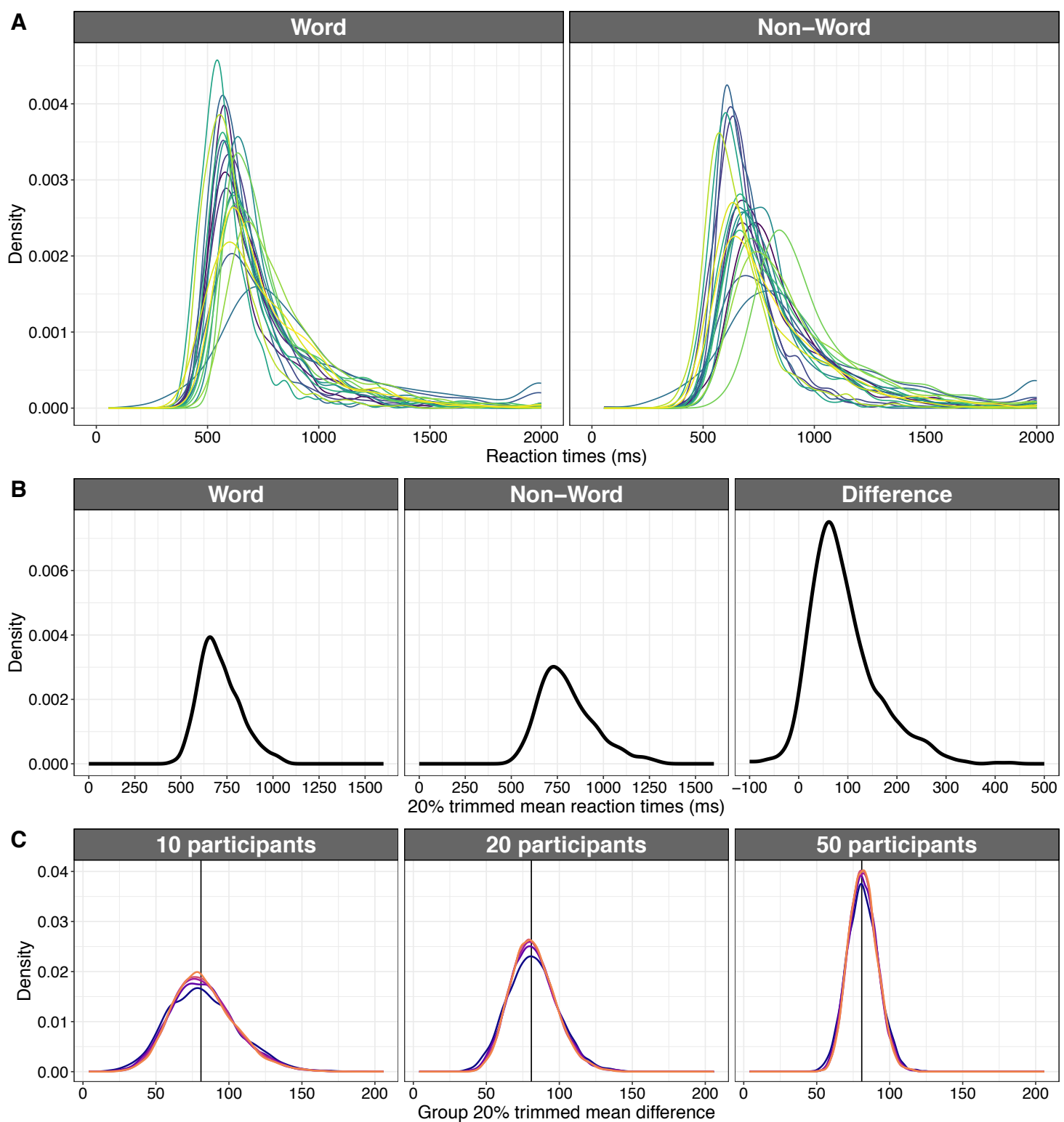

Number of trials

$200-250$



20 participants

$150 \square 200$
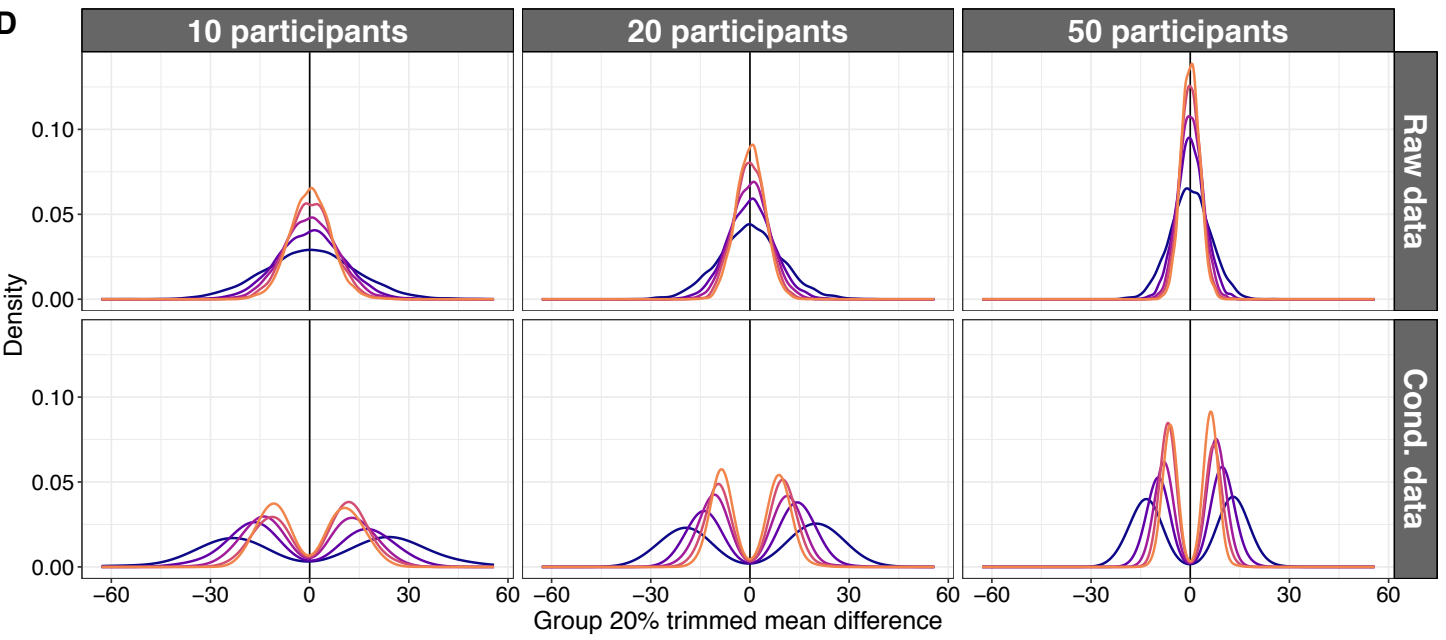
As we saw in the previous examples, by increasing the number of participants, we gain in precision: the distributions get narrower. Interestingly, the number of trials has little effect on the width of the sampling distributions. That's because the effects are large and positive in most participants but vary a lot in magnitude across participants-see the right panel in Figure 8B. Thus, in this situation, it is more beneficial to recruit more participants than to increase the number of trials (Rouder \& Haaf, 2018).

Similarly to what we did in the previous examples, we could compute the number of trials and participants needed to achieve a certain level of estimation precision. But here we use the data to address a different question: what differences could we observe with this type of data and task if there were no difference between conditions? This situation can be simulated by pooling trials across conditions for each participant, and sampling with replacement from that one pool of trials. We proceed by sampling participants with replacement, then for each randomly selected participant, we sample with replacement two sets of random response times from the response times in the two conditions mixed together.

As expected, the sampling distributions are now centred near zero, as on average, in the longrun, we expect zero difference--assuming we're trying to estimate an unbiased quantity (Figure 8D). But the variability across simulated experiments is large, suggesting that if results were selected based on some arbitrary cut-off, researchers could easily fool themselves into reporting false positives of large magnitude. To see what the distributions of results conditional on $p \leq 0.05$ would look like, for each simulation, we performed a group t-test on $20 \%$ trimmed means, a technique that requires some adjustments to the standard t-test equation (Tukey \& McLaughlin, 1963). The conditional distributions look very different to the raw ones (second row of Figure 8D): the distributions are now bimodal with a gap around zero. Based on these distributions, what would the typical result look like? With 10 participants and 50 trials per condition, the median of the absolute group differences is about $24 \mathrm{~ms}$. Among the simulated experiments that produced results with $p \leq 0.05,10 \%$ have differences at least as large as $37 \mathrm{~ms}$, which seems quite substantial for a word recognition task. With 50 participants and 200 trials per condition, the median of the absolute group differences is about $7 \mathrm{~ms}$, and among the experiments with $p \leq 0.05$, 
$10 \%$ produce differences at least as large as $9 \mathrm{~ms}$. So, large sample sizes can strongly damp down the effect sizes of false positives reported in the literature.

\section{Conclusion}

In this article, we saw how simulations can be used to bring perspective to results from single experiments. Using simulations, we can explore potential results not from one experiment, but from thousands of them (sampling distributions). The main outcome of this process is the startling realisation that in many situations, our measurements are so noisy that we should refrain from drawing strong conclusions about them. Instead, we can learn to plan more ambitious experiments from the results of simulations, and develop a healthy scepticism about published results and our own.

Thus, the approach described here contributes to tackling one critical problem faced by psychologists: the over-confidence fuelled by the irrational belief that statistical tests can deliver certainty in the face of measurement noise and sampling variability. More than a crisis of replicability, I would argue that we have a crisis of over-confidence, one that can be tackled not by teaching more stats, but by teaching statistical thinking in the broader sense, but also scientific integrity and humility. In the words of Andrew Gelman (2018): "Forget about getting definitive results from a single experiment; instead embrace variation, accept uncertainty, and learn what you can."

Simulations can be done using synthetic or real data. With data sharing and large studies on the rise, many useful questions can be addressed using data-driven simulations. It's a great time to be a data parasite! Real data have the advantage of providing rich shape information and within participant correlations that can be tricky to simulate. So, given the need for large datasets for running simulations, and the benefits of doing so, this is another reason for scientists to release their data and code.

And we really need more datasets, particularly large multi-site ones. Indeed, in the examples covered above, we only considered variance across trials and participants, but there are many 
more sources of variance we need to account for and try to understand: for instance, variance across stimuli, study parameters, types of equipment, sites, cultures, time of day. Without a good handle on all these sources of variability we face a deep crisis of generalisability (Yarkoni, 2019). Maybe we should have lengthy and passionate discussions about sources of variability and how to improve the precision of our measurements, instead of wasting oxygen discussing $p$ values.

I leave you with an example. At a conference, I saw a presentation in which a group effect of 10 ms between conditions was reported. The researcher went on to discuss the implications of the significant difference $(\operatorname{read} p \leq 0.05)$ for theories of attention. The question session was all about theories, and despite the shaky link between experimental design/analyses/results and the rather abstract theory, no one went Paul Meehl on him. The slide of results had no illustration of the results, not even an ugly bar graph. And nobody asked: how many participants show the effect? How big are the individual effect sizes? How do they compare to results from well-established attentional effects? Nobody said: "About that $10 \mathrm{~ms}$ reaction time group difference, I'd like to show you a sampling distribution-I think you will find it illuminating”. Finally, nobody asked anything about hardware. For instance, if we use a USB keyboard to collect response times, there can be quite a delay, of at least 10-15 ms depending on the model used (Luu, 2017), and there is no guarantee that these delays are constant, especially given that several independent factors influence them (Limmen, 2018). In that context, is a $10 \mathrm{~ms}$ really impressive? Are our measurements precise enough? What other sources of variability originate from our experimental setups?

\section{Acknowledgments}

Thank you toJan Vanhove (@janhove) for comments on an earlier version of this article.

\section{Funding}

The author declares no sources of funding for this work. 


\section{Prior versions}

Some of the examples presented in this article were previously posted in a different format as blog posts covering: sampling distributions of correlations (https://garstats.wordpress.com/2018/06/01/smallncorr/) and what happens when the results are conditioned on $p$ values (https://garstats.wordpress.com/2018/06/22/corrcondpval/), reaction time sampling distributions (https://garstats.wordpress.com/2018/01/24/10000/), and illustrations of estimation precision (https://garstats.wordpress.com/2018/08/27/precision/).

\section{Conflicts of Interest}

The author declares that there were no conflicts of interest with respect to the authorship or the publication of this article.

\section{Disclosures}

All the figures and analyses presented in this article can be reproduced using notebooks in the $R$ programming language ( $\mathrm{R}$ Core Team, 2018), as part of a reproducibility package available on the OSF and on GitHub <https://github.com/GRousselet/sampdist>. All the figures are licensed CG-BY 4.0. Each figure caption ends with the name of the RMarkdown file that can be

used to reproduce it. The main $\mathrm{R}$ packages used to generate the data and to make the figures and notebooks are ggplot2 (Wickham, 2016), cowplot (Wilke, 2017), Cairo (Urbanek \& Horner, 2019), dplyr (Wickham, François, Henry, \& Müller, 2019), Rfast (Papadakis et al., 2019), tibble (Müller \& Wickham, 2018), facetscales (Moreno, 2019), rogme (Rousselet, Pernet, \& Wilcox, 2017), knitr (Xie, 2018), and the essential beepr (Bååth, 2018). 


\section{References}

Bååth, R. (2018). beepr: Easily Play Notification Sounds on any Platform. Retrieved from https://CRAN.Rproject.org/package=beepr

Baguley, T. (2009). Standardized or simple effect size: What should be reported? British Journal of Psychology, 100(3), 603-617. https://doi.org/10.1348/000712608X377117

Baguley, T. (2010). When correlations go bad. The Psychologist, (23), 122-123.

Baguley, T. (2012). Serious Stats: A guide to advanced statistics for the behavioral sciences (2012 edition). Houndmills, Basingstoke, Hampshire England; New York: Palgrave.

Bieniek, M. M., Bennett, P. J., Sekuler, A. B., \& Rousselet, G. A. (2016). A robust and representative lower bound on object processing speed in humans. European Fournal of Neuroscience, 44(2), 1804-1814. https://doi.org/10.1111/ejn.13100

Bland, J. M. (2009). The tyranny of power: Is there a better way to calculate sample size? BMF, 339, b3985. https://doi.org/10.1136/bmj.b3985

Cai, Z., Hahn, A. C., Zhang, W., Holzleitner, I. J., Lee, A. J., DeBruine, L. M., \& Jones, B. C. (2019). No evidence that facial attractiveness, femininity, averageness, or coloration are cues to susceptibility to infectious illnesses in a university sample of young adult women. Evolution and Human Behavior, 40(2), 156-159. https://doi.org/10.1016/j.evolhumbehav.2018.10.002

Dubois, J., Galdi, P., Han, Y., Paul, L. K., \& Adolphs, R. (2018). Resting-State Functional Brain Connectivity Best Predicts the Personality Dimension of Openness to Experience. Personality Neuroscience, 1. https://doi.org/10.1017/pen.2018.8

Ferrand, L., New, B., Brysbaert, M., Keuleers, E., Bonin, P., Méot, A., .. Pallier, C. (2010). The French Lexicon Project: Lexical decision data for 38,840 French words and 38,840 pseudowords. Behavior Research Methods, 42(2), 488-496. https://doi.org/10.3758/BRM.42.2.488

Forstmeier, W., Wagenmakers, E.-J., \& Parker, T. H. (2016). Detecting and avoiding likely false-positive findings - a practical guide. Biological Reviewes, n/a-n/a. https://doi.org/10.1111/brv.12315

Frey, R., Pedroni, A., Mata, R., Rieskamp, J., \& Hertwig, R. (2017). Risk preference shares the psychometric structure of major psychological traits. Science Advances, 3(10), e1701381. https://doi.org/10.1126/sciadv.1701381 
Gelman, A. (2018). The Failure of Null Hypothesis Significance Testing When Studying Incremental Changes, and What to Do About It. Personality and Social Psychology Bulletin, 44(1), 16-23. https://doi.org/10.1177/0146167217729162

Gelman, A., \& Carlin, J. (2014). Beyond Power Calculations: Assessing Type S (Sign) and Type M (Magnitude) Errors. Perspectives on Psychological Science, 9(6), 641-651. https://doi.org/10.1177/1745691614551642

Gelman, A., \& Weakliem, D. (2009). Of Beauty, Sex and Power: Too little attention has been paid to the statistical challenges in estimating small effects. American Scientist, 97(4), 310-316. Retrieved from JSTOR.

Gigerenzer, G., \& Marewski, J. N. (2015). Surrogate Science: The Idol of a Universal Method for Scientific Inference. Journal of Management, 41(2), 421-440. https://doi.org/10.1177/0149206314547522

Greenland, S., Senn, S. J., Rothman, K.J., Carlin, J. B., Poole, C., Goodman, S. N., \& Altman, D. G. (2016). Statistical tests, $\mathrm{P}$ values, confidence intervals, and power: A guide to misinterpretations. European fournal of Epidemiology, 31(4), 337-350. https://doi.org/10.1007/s10654-016-0149-3

Howell, D. C. (2013). Statistical Methods for Psychology (8th edition). Wadsworth Cengage Learning.

Ioannidis, J. P. A. (2008). Why most discovered true associations are inflated. Epidemiology (Cambridge, Mass.), 19(5), 640-648. https://doi.org/10.1097/EDE.0b013e31818131e7

Jaeger, T. F. (2008). Categorical Data Analysis: Away from ANOVAs (transformation or not) and towards Logit Mixed Models. Fournal of Memory and Language, 59(4), 434-446. https://doi.org/10.1016/j.jml.2007.11.007

Kruschke, J. K. (2014). Doing Bayesian Data Analysis (2nd Edition). Academic Press.

Limmen, C. (2018, May 31). What influences keyboard input speed. Retrieved 30 May 2019, from Wooting developer blog website: https://blog.wooting.nl/what-influences-keyboard-speed/

Loken, E., \& Gelman, A. (2017). Measurement error and the replication crisis. 355(6325), 584-585. https://doi.org/10.1126/science.aal3618

Luu, D. (2017). Keyboard latency. Retrieved 30 May 2019, from Danluu.com website: https://danluu.com/keyboard-latency/

Maxwell, S. E., Kelley, K., \& Rausch,J. R. (2008). Sample size planning for statistical power and accuracy in parameter estimation. Annual Review of Psychology, 59, 537-563.

https://doi.org/10.1146/annurev.psych.59.103006.093735 
McShane, B. B., Gal, D., Gelman, A., Robert, C., \& Tackett, J. L. (2019). Abandon Statistical Significance. The American Statistician, 73(sup1), 235-245. https://doi.org/10.1080/00031305.2018.1527253

Moreno, S. O. (2019). facetscales: Facet_grid with different scales per facet. Retrieved from http://github.com/zeehio/facetscales

Morey, R. D. (2018, January 3). When the statistical tail wags the scientific dog. Retrieved 4 December 2019, from Medium website: https://medium.com/@richarddmorey/when-the-statistical-tail-wags-the-scientific-dogd09a9fla7c63

Müller, K., \& Wickham, H. (2018). tibble: Simple Data Frames. Retrieved from https://CRAN.Rproject.org/package $=$ tibble

Nowak, L. G., \& Bullier, J. (1997). The Timing of Information Transfer in the Visual System. In K. S. Rockland, J. H. Kaas, \& A. Peters (Eds.), Extrastriate Cortex in Primates (pp. 205-241). https://doi.org/10.1007/978-14757-9625-4_5

Papadakis, M., Tsagris, M., Dimitriadis, M., Fafalios, S., Tsamardinos, I., Fasiolo, M., ... Chatzipantsiou, C. (2019). Rfast: A Collection of Efficient and Extremely Fast R Functions. Retrieved from https://CRAN.Rproject.org/package $=$ Rfast

Peters, G.-J., \& Crutzen, R. (2017). Knowing how effective an intervention, treatment, or manipulation is and increasing replication rates: Accuracy in parameter estimation as a partial solution to the replication crisis. https://doi.org/10.31234/osf.io/cjsk2

Pietschnig, J., Penke, L., Wicherts, J. M., Zeiler, M., \& Voracek, M. (2015). Meta-analysis of associations between human brain volume and intelligence differences: How strong are they and what do they mean? Neuroscience E Biobehavioral Reviews, 57, 411-432. https://doi.org/10.1016/j.neubiorev.2015.09.017

Plonsky, L., \& Oswald, F. L. (2014). How Big Is "Big"? Interpreting Effect Sizes in L2 Research. Language Learning, 64(4), 878-912. https://doi.org/10.1111/lang.12079

R Core Team. (2018). R: A Language and Environment for Statistical Computing. Retrieved from https://www.Rproject.org/

Ritchie, S. J., Cox, S. R., Shen, X., Lombardo, M. V., Reus, L. M., Alloza, C., .. Deary, I. J. (2018). Sex Differences in the Adult Human Brain: Evidence from 5216 UK Biobank Participants. Cerebral Cortex, 28(8), 2959-2975. https://doi.org/10.1093/cercor/bhy109 
Rothman, K. J., \& Greenland, S. (2018). Planning Study Size Based on Precision Rather Than Power. Epidemiology, 29(5), 599. https://doi.org/10.1097/EDE.0000000000000876

Rouder, J. N., \& Haaf, J. M. (2018). Power, Dominance, and Constraint: A Note on the Appeal of Different Design Traditions. Advances in Methods and Practices in Psychological Science, 1(1), 19-26. https://doi.org/10.1177/2515245917745058

Rouder, J. N., \& Province, J. M. (2019). Bayesian Hierarchical Models In Psychological Science: A Tutorial. In Frontiers of Cognitive Psychology. New Methods in Cognitive Psychology (1st ed., pp. 32-66). Routledge.

Rousselet, G. A., Pernet, C. R., \& Wilcox, R. R. (2017). Beyond differences in means: Robust graphical methods to compare two groups in neuroscience. European Fournal of Neuroscience, 46(2), 1738-1748. https://doi.org/10.1111/ejn.13610

Rousselet, G. A., Pernet, G. R., \& Wilcox, R. R. (2019). A practical introduction to the bootstrap: A versatile method to make inferences by using data-driven simulations [Preprint]. Retrieved from PsyArXiv website: https://doi.org/10.31234/osf.io/h8ft7

Rousselet, G. A., \& Wilcox, R. R. (2019). Reaction times and other skewed distributions: Problems with the mean and the median [Preprint]. https://doi.org/10.31234/osf.io/3y54r

Schönbrodt, F. D., \& Perugini, M. (2013). At what sample size do correlations stabilize? Fournal of Research in Personality, 47(5), 609-612. https://doi.org/10.1016/j.jrp.2013.05.009

Shaffer, J. P. (2002). Multiplicity, directional (type III) errors, and the null hypothesis. Psychological Methods, 7(3), 356369.

Smaldino, P. E., \& McElreath, R. (2016). The natural selection of bad science. Royal Society Open Science, 3(9), 160384. https://doi.org/10.1098/rsos.160384

Trafimow, D., \& MacDonald, J. A. (2017). Performing Inferential Statistics Prior to Data Collection. Educational and Psychological Measurement, 77(2), 204-219. https://doi.org/10.1177/0013164416659745

Trafimow, D., Wang, T., \& Wang, C. (2018). Means and standard deviations, or locations and scales? That is the question! New Ideas in Psychology, 50, 34-37. https://doi.org/10.1016/j.newideapsych.2018.03.001

Trafimow, D., Wang, T., \& Wang, C. (2019). From a Sampling Precision Perspective, Skewness Is a Friend and Not an Enemy! Educational and Psychological Measurement, 79(1), 129-150.

https://doi.org/10.1177/0013164418764801 
Tukey, J. W., \& McLaughlin, D. H. (1963). Less Vulnerable Confidence and Significance Procedures for Location Based on a Single Sample: Trimming/Winsorization 1. Sankhyā: The Indian fournal of Statistics, Series A (19612002), 25(3), 331-352. Retrieved from JSTOR.

Urbanek, S., \& Horner, J. (2019). Cairo: R Graphics Device using Cairo Graphics Library for Creating High-Quality Bitmap (PNG, JPEG, TIFF), Vector (PDF, SVG, PostScript) and Display (X11 and Win32) Output. Retrieved from https:/ CRAN.R-project.org/package=Cairo

Vul, E., Harris, C., Winkielman, P., \& Pashler, H. (2009a). Puzzlingly High Correlations in fMRI Studies of Emotion, Personality, and Social Cognition. Perspectives on Psychological Science: A fournal of the Association for Psychological Science, 4(3), 274-290. https://doi.org/10.1111/j.1745-6924.2009.01125.x

Vul, E., Harris, C., Winkielman, P., \& Pashler, H. (2009b). Reply to Comments on 'Puzzlingly High Correlations in fMRI Studies of Emotion, Personality, and Social Cognition'. Perspectives on Psychological Science: A Journal of the Association for Psychological Science, 4(3), 319-324. https://doi.org/10.1111/j.1745-6924.2009.01132.x

Wickham, H. (2016). ggplot2: Elegant Graphics for Data Analysis (2nd ed.). Retrieved from https://www.springer.com/gb/book/9783319242750

Wickham, H., François, R., Henry, L., \& Müller, K. (2019). dplyr: A Grammar of Data Manipulation. Retrieved from https:/ / CRAN.R-project.org/package=dplyr

Wilcox, R. R. (2017). Introduction to Robust Estimation and Hypothesis Testing (4th edition). Academic Press.

Wilcox, R. R., \& Rousselet, G. A. (2018). A Guide to Robust Statistical Methods in Neuroscience. Current Protocols in Neuroscience, 82(1), 8.42.1-8.42.30. https://doi.org/10.1002/cpns.41

Wilke, C. O. (2017). cowplot: Streamlined Plot Theme and Plot Annotations for 'ggplot2'. Retrieved from https://CRAN.Rproject.org/package $=$ cowplot

Xie, Y. (2018). knitr: A General-Purpose Package for Dynamic Report Generation in R. Retrieved from https://yihui.name/knitr/

Yarkoni, T. (2009). Big Correlations in Little Studies: Inflated fMRI Correlations Reflect Low Statistical PowerCommentary on Vul et al. (2009). Perspectives on Psychological Science: A Journal of the Association for Psychological Science, 4(3), 294-298. https://doi.org/10.1111/j.1745-6924.2009.01127.x

Yarkoni, T. (2019). The Generalizability Crisis [Preprint]. https://doi.org/10.31234/osf.io/jqw35 
Zhang, W., Hahn, A. G., Gai, Z., Lee, A. J., Holzleitner, I. J., DeBruine, L. M., \& Jones, B. C. (2018). No evidence that facial width-to-height ratio (fWHR) is associated with women's sexual desire. PLOS ONE, 13(7), e0200308. https://doi.org/10.1371/journal.pone.0200308 